\title{
Resíduo Institucional: limpeza pública e conservação na UNICAMP e minimização de resíduo na FEC
}

\author{
Institutional waste: public cleaning and conservation at UNICAMP and waste \\ minimization in FEC
}

\begin{abstract}
Eglé Novaes Teixeira
Graduada em Eng. Civil (1978), mestre em Eng. Agrícola (1985) pela UNICAMP, Especialista em Eng. Sanitária pelo IHE (Delft) Netherland) e Dra em Eng. Civil pela USP (1993). Profa. na FEC/UNICAMP, trabalha com gerenciamento de resíduo sólido.
\end{abstract}

\section{José Benedito de Castro Henrique}

Graduado em Economia (1990), especialista em Gestão Ambiental (2005) e mestre em Engenharia Civil (2008), todos pela UNICAMP. É Diretor de Limpeza Urbana da Prefeitura da UNICAMP, desde 2001.

Martina Barbosa

Graduada em Tecnologia em Saneamento Ambiental (2005), mestre em Engenharia Civil (2007) pela UNICAMP. Atualmente, doutoranda em Engenharia Civil na FEC/UNICAMP.

Endereço:* Faculdade de Engenharia Civil, Arquitetura e Urbanismo - FEC - UNICAMP. Avenida Albert Einstein, 951,Cidade Universitária "Zeferino Vaz”. Caixa Postal 6021. CEP: 13083-852 - Campinas - SP.

\section{RESUMO}

Um dos grandes desafios atuais é o que fazer com a enorme quantidade de resíduo produzido diariamente. No Brasil, buscase por solução para essa questão e a gestão adequada de resíduo sólido é essencial para compor uma solução para a transformação do atual modelo de serviço público de limpeza urbana em um sistema integrado, buscando a minimização. A determinação da composição gravimétrica e os fatores que influenciam na geração de resíduo sólido urbano são de grande importância em todas as ações que compõem os serviços de limpeza pública. Os resíduos provenientes da limpeza do Campus e de uma Unidade de Ensino foram caracterizados e a ampliação do conhecimento sobre a geração desses resíduos e dos serviços de limpeza urbana do Campus, permitiu conhecer e melhorar a gestão desses serviços.

Palavras-chave: resíduo sólido institucional; resíduos sólidos domésticos; limpeza pública; caracterização; minimização.

\section{ABSTRACT}

One of the greatest challenges nowadays is what to do with the huge quantity of waste generated daily. In Brazil, there is a search for a solution for this problem. The appropriate solid waste management is essential to compose the solution for the transformation of the public service of urban cleaning current model in an integrated system, considering the minimization. The determination of the gravimetrical composition and the factors of the urban solid waste generation influence are important in the public cleaning services planning and operating phases.
The public cleaning waste at UNICAMP Campus and a School waste were characterized, and the enlargement of the knowledge about the waste generation and the urban cleaning service practices on Zeferino Vaz University Campus allowed to know and to improve the management of these services.

Key-words: institutional solid waste; domestic solid waste; public cleaning; characterization; minimization.

\section{INTRODUÇÃO}

Junto com a população e a industrialização, a produção de resíduo cresceu, causando, degradação ambiental e sérios problemas à qualidade de vida do homem. A questão da produção e da destinação adequada do resíduo nos grandes centros urbanos é um problema complexo que desafia a sociedade a encontrar, com urgência, soluções para a sua destinação adequada e para sua minimização.

No Brasil, a sociedade está à procura de soluções para enfrentar a questão de geração e destinação adequada do resíduo. O conhecimento detalhado dos serviços de limpeza urbana, englobando a coleta, tratamento e destinação final e a minimização, despontam como uma das principais ações para a composição de uma solução.

As instituições escolares, primordiais no processo de formação dos indivíduos, aparecem como importantes locais de ação no desenvolvimento de programas que visem à melhor gestão do resíduo, por meio de sua minimização, por seu papel na formação de agentes multiplicadores. Há a necessidade de implantação de novos projetos de sensibilização em relação à problemática do resíduo sólido direcionada para o envolvimento da comunidade escolar.

Neste trabalho efetuou-se a caracterização do resíduo de conservação e limpeza, do Campus Cidade Universitária "Zeferino Vaz" da Universidade Estadual de Campinas (UNICAMP), detalhou-se a logística de manejo, ampliouse o conhecimento sobre a geração e o gerenciamento deste resíduo, servindo como apoio à gestão e ao gerenciamento de resíduo sólido urbano de outros campi universitários e desenvolveu-se, na Faculdade de Engenharia Civil, Arquitetura e Urbanismo (FEC) da UNICAMP, um programa de minimização de resíduo sólido doméstico (HENRIQUE, 2008 e BARBOSA, 2007).

Considerando as dificuldades de envolvimento, sensibilização e conscientização da comunidade em relação a programas de gestão e minimização de resíduo e também de mudança de hábitos, neste trabalho, avaliaram-se os pontos positivos e a serem melhorados no sistema de limpeza e conservação da UNICAMP; o potencial para ampliação das atividades de minimização de resíduo na Universidade; e, também, a implantação e a eficiência do programa de minimização realizado na FEC.

Destaca-se que este trabalho está inserido no programa de 


\section{Resíduo Sólido da Prefeitura da UNICAMP. \\ 2. OBJETIVOS}

- Caracterizar e mensurar o resíduo sólido urbano de conservação e limpeza gerenciado pelo serviço de limpeza urbana do Campus;

- Propor ações que otimizem o planejamento e o gerenciamento deste resíduo;

- Avaliar o potencial de minimização do resíduo sólido doméstico gerado um uma unidade de ensino da UNICAMP (FEC); e,

- Implantar um programa de minimização de resíduo sólido doméstico na FEC.

\section{METODOLOGIA}

Está dividida em: definição das datas de caracterização; avaliação dos serviços de limpeza pública e de conservação da UNICAMP; e; implantação do programa de minimização do resíduo sólido doméstico gerado na FEC.

\subsection{Definição das datas de caracterização}

O plano de amostragem escolhido foi o de amostragem sistemática, porém, sem um intervalo de tempo definido, pois este varia de acordo com os fatores que interferem na geração de resíduo. Dessa forma, foram definidos os períodos de caracterização contem-plando os dias da semana, semanas dos meses e as estações do ano.

Na elaboração do calendário de caracterização foram evitados feriados e eventos especiais da Universidade (Universidade de Portas Abertas, Semana da Engenharia Civil, Semana da Arquitetura, etc.) entre ou-tros acontecimentos, que poderiam descaracterizar a produção média de resíduo.

O cronograma de caracterização do Resíduo de Conservação e Limpeza foi elaborado para um período de 12 meses, totalizando 16 semanas. Já para o resíduo sólido doméstico da FEC foram realizadas 21 semanas, no decorrer de 18 meses.

\subsection{Avaliação dos serviços de limpeza pública e de conserva- ção da UNICAMP}

A metodologia para avaliação foi composta por: composição do resíduo sólido gerado em áreas comuns da UNICAMP; definição da composição dos itens caracterizados; avaliação do potencial de minimização do resíduo; e, logística de caracterização do resíduo de conservação e limpeza.

\subsubsection{Composição do resíduo sólido gerado nas áreas co- muns da UNICAMP}

O resíduo sólido gerado nas áreas comuns foi proveniente de: feira regular: ocorre às terças e sextas-feiras no estacionamento do Centro de Saúde da Comunidade e às quartas-feiras na Reitoria; e, feiras de artesanato e quitutes que ocorrem no Ciclo Básico, às quintas-feiras, e na Área Médica, em datas variadas; e, serviços de varrição de ruas; limpeza de lixeiras e grades de lixo; e, coleta de detritos de gramados.

Estes resíduos foram denominados:

- Resíduo de Feira: resíduo proveniente de feiras;

- Resíduo de Limpeza: resíduo proveniente dos serviços de varrição de ruas; limpeza de lixeiras e grades de lixo; e, coleta de detritos em gramado; e,

- Resíduo de Conservação e Limpeza: resíduo de feira somado ao resíduo de limpeza.

\subsubsection{Definição da composição dos itens caracterizados}

Os itens caracterizados foram separados para viabilizar o diagnóstico do material que compõe este resíduo, possibilitando a otimização de seu gerenciamento.

No Resíduo de Conservação e Limpeza buscou-se potencial de material reciclável contido no mesmo, para poder avaliar a possibilidade da expansão do Programa de Coleta Seletiva para as áreas comuns do Campus.

Os itens analisados no resíduo de conservação e limpeza foram: papel branco reutilizável (não utilizado ou utilizado apenas de um lado), somente foram consideradas as folhas com pelo menos $50 \%$ do tamanho de uma folha A4; papel branco (utilizado dos dois lados), ou com menos de 50\% do tamanho de uma folha de papel A4; papel misto (co-lorido/pardo/revistas); papelão; jornal; vidro; plástico; metal (alumínio, cobre e bronze); ferro (sucata ferrosa); não reciclável (pilhas, baterias, embalagens Tetra Pak, embalagens compostas de plástico e papel ou plástico e alumínio, borracha, material muito sujo); orgânico (restos de comida e frutas); patogênico ( papel higiênico, remédios, etc); fraldas; e, varrição (folhas, galhos, pedras, terra e poeira).

Tanto o Resíduo de Feira como o resíduo de Limpeza foram caracterizados. O resíduo foi pesado, triado, colocado em bombonas específicas para cada categoria de separação e, então, pesados.

\subsubsection{Avaliação do potencial de minimização do resíduo de conservação e limpeza}

Considerou-se para avaliação do potencial de minimização de resíduo de conservação e limpeza apenas o papel branco. Este material foi escolhido em função de sua geração ser predominante nas atividades administrativas da Universidade.

Determinou-se a quantidade de papel branco reutilizável, ou seja, fo-lhas descartadas com ambos ou um de seus lados em branco. Com o potencial de minimização podem ser desenvolvidas estratégias que visem à redução do desperdício deste material. Somente foram consideradas folhas com, pelo menos, $50 \%$ do tamanho de uma folha de papel A4.

\subsection{Implantação do Programa de Minimização do Resíduo Sólido Doméstico da FEC}

Este item foi composto por: caracterizações feitas antes e após a implantação do Programa; avaliação do potencial de minimiza- ção

3.3

Pro

Par

ger

doz

Ap

car:

tre

o $\mathrm{m}$

par

pla

Du

tific

fina

rac

foi

Os

plá:

liza

lad

toa

plá

láv

hei$$
\text { out }
$$

A s
tuin

\section{3.}

\section{$\mathrm{Na}$}

min$$
\text { min }
$$$$
\text { teri }
$$

pot

$\mathrm{Na}$

- $\mathrm{re}$

- $\mathrm{p}$ 
ção; e, elaboração e implantação do Programa.

3.3.1 Caracterizações antes e após a implantação do Programa

Para conhecer a composição do resíduo sólido doméstico gerado na FEC e a eficiência do programa foram realizadas doze caracte-rizações antes de seu início.

Após a implantaçãZo do Programa foram realizadas nove caracterizações, possibilitando, assim, uma comparação entre a quantidade e qualidade de resíduo gerado antes e após o mesmo. A metodologia de caracterização utilizada, tanto para as caracte-rizações realizadas antes quanto após a implantação do programa, foi a mesma.

Durante as semanas de coleta, o resíduo da FEC foi identificado e colocado em um local específico para coleta. No final de cada dia, o resíduo foi coletado e levado ao barracão da Divisão de Meio Ambiente da UNICAMP, onde foi caracterizado.

Os sacos plásticos foram, inicialmente, pesados e a massa anotada, constituindo a massa total. Em seguida, os sacos plásticos foram abertos e o conteúdo separado nas seguintes categorias: papel branco não utilizado; papel branco utilizado apenas de um lado; papel branco utilizado de dois lados; papel pardo envelopes; papel pardo em geral; papel toalha; outros papéis recicláveis; e, outros papéis não recicláveis; plástico reciclável; plástico não reciclável; e, copos plásticos descartáveis; vidro reciclável; e, vidro não reciclável; metal; patogênico (papel higiênico utilizado nos banheiros); material orgânico desperdício; varrição; e, material orgânico outros; material misto; embalagens Tetra Pak; e, outros tipos de materiais.

A seguir, cada categoria foi pesada e as massas anotadas constituindo a massa por categoria.

\subsubsection{Avaliação do potencial de minimização}

Na FEC foi feita uma avaliação mais detalhada do potencial minimização, diferentemente do resíduo de Limpeza e Conservação, já que o objetivo foi a implantação de um programa de minimização. A partir da análise dos dados obtidos nas caracterizações anteriores à implantação do Programa, foi avaliado o potencial de minimização do resíduo sólido gerado na FEC:

Na determinação do potencial de minimização foram adotadas as considerações:

- redução na fonte: papel branco não utilizado; papel toalha; copos descartáveis; e, material orgânico desperdício;

- reutilização: papel branco utilizado apenas de um lado; e, envelopes;

- reciclagem: papel branco utilizado dos dois lados; papel pardo; outros papeis recicláveis; vidro reciclável; metal ferroso; metal não ferroso; plástico reciclável; e, Tetra Pak; e,

- aterro: papel não reciclável; vidro não reciclável; plástico não reciclável; patogênico; material orgânico outros varrição; perigoso; misto; e, outro tipo de resíduo não reciclável. Estes dados forneceram subsídios para a elaboração do Programa de Minimização de Resíduo Sólido da FEC.
3.3.3 Elaboração e implantação do programa de minimização de resíduo sólido doméstico

Foi elaborado um programa de minimização, com base no potencial de minimização do resíduo sólido. Este programa foi baseado nos conceitos de redução na fonte, reutilização e reciclagem.

O princípio norteador do programa foi que este deveria ser considerado nas decisões cotidianas a serem tomadas por todos os membros da comunidade. Assim, foi requerido o envolvimento de todos: toda a comunidade é responsável por minimizar sua geração de resíduo sólido por meio de práticas corretas de manejo e de destinação do material para a coleta seletiva. A comunidade FEC é formada por um público diversificado.

Neste programa buscou-se sensibilizar a comunidade quanto aos pro-blemas resultantes do desperdício e quanto à má destinação do material passível de ser reciclado, buscando a conscientização em relação ao tema.

Na sensibilização da comunidade universitária foram abordados conteúdos que refletem as causas da existência da grande geração de resíduo, assim como suas consequências para a saúde e o ambiente.

A implantação e divulgação do Programa foram feitas por meio de pa-lestras em salas de aulas, boletins de divulgação, reuniões informativas destacando assuntos como desperdício, coleta seletiva e minimização de resíduo sólido.

\section{Resultados e discussões}

Este item está dividido em: datas de caracterização; resíduo dos serviços de limpeza pública e de conservação gerados nas áreas comuns da UNICAMP; e, programa de minimização do resíduo sólido gerado na FEC.

\subsection{Datas de caracterização}

Na Tabela. 4.1 são mostradas as datas das caracterizações realizadas no resíduo de conservação e limpeza gerado nas áreas comuns da UNICAMP e nas Tabelas 4.2 e 4.3, antes e após a implantação do programa de minimização de resíduo sólido doméstico da FEC.

Tabela 4.1 Datas das caracterizações do resíduo de conser-

\begin{tabular}{|c|c|c|c|}
\hline Período & Semana do mês & Estação do ano & Caracterização \\
\hline De $30 / 01$ a $03 / 02 / 06$ (férias) & $1^{\underline{a}}$ & Verão & 5 \\
\hline De 13 a 17/02/06 (férias) & $3^{\mathrm{a}}$ & Verão & 7 \\
\hline De 06 a $10 / 03 / 06$ & $2^{\mathrm{a}}$ & Verão & 10 \\
\hline De 27 a $31 / 03 / 06$ & $5^{\mathrm{a}}$ & Outono & 13 \\
\hline De 24 a 28/04/06 & $4^{\mathrm{a}}$ & Outono & 17 \\
\hline De 15 a $19 / 05 / 06$ & $3^{\mathrm{a}}$ & Outono & 20 \\
\hline De 05 a $09 / 06 / 06$ & 1 & Outono & 23 \\
\hline De 03 a 07/07/06 & $1^{\mathrm{a}}$ & Inverno & 27 \\
\hline De 14 a $18 / 08 / 06$ & $3^{\mathrm{a}}$ & Inverno & 33 \\
\hline De 21 a 25/08/06 & $4^{\mathrm{a}}$ & Inverno & 34 \\
\hline De 11 a $15 / 09 / 06$ & $2^{\mathrm{a}}$ & Inverno & 37 \\
\hline De 25 a 29/09/06 & $4^{\mathrm{a}}$ & Primavera & 39 \\
\hline De 16 a $20 / 10 / 06$ & $3^{\mathrm{a}}$ & Primavera & 42 \\
\hline De 06 a $10 / 11 / 06$ & $2^{\mathrm{a}}$ & Primavera & 45 \\
\hline De $27 / 11$ a $01 / 12 / 06$ & $5^{\mathrm{a}}$ & Primavera & 48 \\
\hline De 22 a 26/01/06 (férias) & $4^{\mathrm{a}}$ & Verão & 56 \\
\hline
\end{tabular}


vação e limpeza da UNICAMP

Tabela 4.2 Datas das caracterizações realizadas antes da implantação do programa de minimização de resíduo

\begin{tabular}{lclc}
\multicolumn{1}{c}{ Período } & Semana do mês & \multicolumn{1}{c}{ Estação do ano } & Caracterização \\
De 27/06/05 a 02/07/05 & $4^{\underline{\underline{a}}}$ & Inverno & 1 \\
De 25 a 30/07/05 (férias) & $4^{\underline{a}}$ & Inverno & 2 \\
De 08 a 13/08/05 & $1^{\underline{\underline{a}}}$ & Inverno & 3 \\
De 22 a 27/08/05 & $3^{\underline{a}}$ & Inverno & 4 \\
De 12 a 17/09/05 & $2^{\underline{a}}$ & Inverno & 5 \\
De 03 a 08/10/05 & $1^{\underline{\underline{a}}}$ & Primavera & 6 \\
De 17 a 22/10/05 & $3^{\underline{a}}$ & Primavera & 7 \\
De 07 a 12/11/05 & $2^{\underline{a}}$ & Primavera & 8 \\
De 23 a 28/01/06 (férias) & $4^{\underline{a}}$ & Verão & 9 \\
De 01 a 08/02/06 (férias) & $1^{\underline{a}}$ & Verão & 10 \\
De 13 a 18/02/06 (férias) & $2^{\underline{a}}$ & Verão & 11 \\
De 20 a 25/02/06 (férias) & $3^{\underline{a}}$ & Verão & 12
\end{tabular}

sólido doméstico

Tabela 4.3 Datas das caracterizações realizadas após a implantação do programa de minimização de resíduo

\begin{tabular}{lclc}
\multicolumn{1}{r}{ Período } & Semana do mês & Estação do ano & Caracterização \\
De 03/04 a 08/04/06 & $1^{\underline{\underline{a}}}$ & Outono & 13 \\
De 24 a 29/04/06 & $4^{\underline{a}}$ & Outono & 14 \\
De 15 a 20/05/06 & $3^{\underline{a}}$ & Outono & 15 \\
De 08 a 14/06/06 & $2^{\underline{a}}$ & Outono & 16 \\
De 03 a 08/07/06 & $1^{\underline{a}}$ & Inverno & 17 \\
De 17 a 22/07/06 (férias) & $3^{\underline{a}}$ & Inverno & 18 \\
De 14 a 19/08/06 & $3^{\underline{a}}$ & Inverno & 19 \\
De 28/08 a 02/09/06 & $4^{\underline{a}}$ & Inverno & 20 \\
De 11 a 16/09/06 & $2^{\underline{a}}$ & Inverno & 21
\end{tabular}

sólido doméstico

4.2 Resíduo de conservação e limpeza gerado nas áreas comuns da UNICAMP

O resíduo de conservação e limpeza gerado nas áreas comuns da UNICAMP é composto pelos resíduos de feira; e, limpeza.

\subsubsection{Resíduo de Feira}

Na Tabela. 4.3 são apresentados os resultados das caracterizações do Resíduo de Feira gerado na UNICAMP.

\begin{tabular}{|c|c|c|c|c|c|c|c|c|c|c|c|c|c|c|c|}
\hline Estação & $\begin{array}{ll}\mathrm{Pp} & \text { bco } \\
\text { reutilizável }\end{array}$ & Pp bco & Pp misto & Papelão & Jornal & Vidro & Plástico & Metal & Ferro & Fraldas & Patogênicd & ñ reciclável & Varrição & Total & $\%$ \\
\hline Verão & 0,00 & 6,96 & 18,05 & 5,10 & 1,40 & 6,52 & 36,60 & 0,80 & 3,19 & 41,10 & 0,00 & 139,10 & 102,78 & 361,59 & 38,67 \\
\hline Outono & 0,00 & 15,79 & 17,05 & 2,60 & 0,00 & 0,70 & 34,10 & 0,99 & 0,00 & 0,90 & 0,00 & 93,04 & 0,00 & 165,17 & 17,66 \\
\hline Inverno & 0,00 & 13,05 & 4,40 & 4,7 & 0,00 & 0,10 & 33,10 & 0,235 & 0,00 & 0,00 & 0,00 & 87,90 & 44,00 & 187,49 & 20,05 \\
\hline Primavera & 0,00 & 14,90 & 5,20 & 4,20 & 0,90 & 4,60 & 49,00 & 0,32 & 0,00 & 0,00 & 0,00 & 141,70 & 0,00 & 220,82 & 23,62 \\
\hline Total & 0,00 & 50,70 & 44,70 & 16,60 & 2,30 & 11,92 & 152,80 & 2,34 & 3,19 & 42,00 & 0,00 & 461,74 & 146,78 & 935,06 & \\
\hline$\%$ & 0,00 & 5,42 & 4,78 & 1,77 & 0,25 & 1,27 & 16,34 & 0,25 & $\overline{0,34}$ & 4,49 & 0,00 & 49,38 & 15,70 & & 100,00 \\
\hline
\end{tabular}

Tabela 4.3 Resíduo de Feira gerado no Campus Cidade Universitária "Zeferino Vaz" da UNICAMP, por estação do ano (kg).

Observa-se na Tabela. 4.3, que a maior geração de resíduo de feira ocorreu no verão e depois na primavera. Ressalta-se que a maior presença de pessoas nas feiras foi nos períodos mais quentes do ano. A média de geração do Resíduo de Feira foi de $58,5 \mathrm{~kg}$.

Na Tabela 4.4 observa-se a distribuição do Resíduo de Feira entre as semanas de caracterização e a que semana do mês se

refere. A distribuição foi por categorias de massa $(\mathrm{kg})$, variando cada categoria em $10 \mathrm{~kg}$, aproximadamente $1 \%$ do total de resíduo

\begin{tabular}{c|c|c|c}
\hline \multicolumn{2}{c|}{ Categorias (kg) } & $\begin{array}{c}\text { Semana de } \\
\text { caracterização }\end{array}$ & Semana do mês \\
\hline 1 & $21-30$ & 20 & $3^{\underline{a}}$ \\
\hline 2 & $31-40$ & 13 e 34 & $5^{\underline{a}}$ e $4^{\underline{a}}$ \\
\hline 3 & $41-50$ & 5,23 e 37 & $1^{\underline{a}}, 1^{\underline{a}}$ e $2^{\underline{a}}$ \\
\hline $\mathbf{4}$ & $\mathbf{5 1 - 6 0}$ & 51 e $\mathbf{6 0}$ & $4^{\underline{a}}$ e 4 \\
\hline 5 & $61-70$ & 45 e 48 & $2^{\underline{a}}$ e $5^{\underline{a}}$ \\
\hline 6 & $71-80$ & $10,27,33$ e 42 & $2^{-}, 1^{\underline{a}}, 3^{-}$e $3^{\underline{a}}$ \\
\hline 7 & $81-90$ & 7 e 17 & $3^{\underline{a}}$ e $4^{\underline{a}}$ \\
\hline
\end{tabular}

caracterizado.

\section{Tabela 4.4 Distribuição do Resíduo de Feira porcategorias de massa}

Verifica-se na Tabela. 4.4 que a média de geração de resíduo, esteve na 4a categoria, com: duas 4a semanas. Abaixo da categoria da média, foram 6 semanas, sendo: duas 1a, uma 2a, uma 3a, uma 4 a e uma 5a semana. Acima da categoria da média, 8 semanas, sendo: uma 1a, duas $2 \mathrm{a}$, três $3 \mathrm{a}$, uma 4a e uma 5a semana. Ao se analisar a influência de fatores externos neste resíduo,
4.2

\section{$\mathrm{Na}$}

\section{No}

do $t$

iten

\section{$\mathrm{Na}$}

refe

$$
\text { de } \mathrm{r}
$$


4.2.2 Resíduo de Limpeza

\begin{tabular}{c|c|c|c|c|c|c|c|c|c|c|c|c|c|c|c}
\hline & $\begin{array}{l}\text { Pp bco } \\
\text { Estação } \\
\text { reutilizável }\end{array}$ & Pp bco & Pp misto & Papelão & Jornal & Vidro & Plástico & Metal & Ferro & Fraldas & Patogênico & ñ reciclável & Varrição & Total & $\%$ \\
\hline Verão & 1,06 & 64,36 & 94,35 & 73,49 & 1,10 & 67,72 & 328,46 & 12,26 & 15,69 & 2,70 & 57,18 & 580,76 & 1349,33 & 2648,47 & 24,29 \\
\hline Outono & 9,11 & 57,04 & 82,80 & 64,05 & 0,90 & 86,01 & 299,50 & 14,78 & 2,21 & 0,00 & 35,75 & 583,09 & 1186,76 & 2422,00 & 22,21 \\
\hline Inverno & 0,80 & 55,45 & 67,40 & 54,45 & 0,00 & 48,30 & 187,20 & 2,85 & 0,00 & 8,3 & 16,88 & 388,40 & 2010,72 & 2840,75 & 26,05 \\
\hline Primavera & 0,00 & 59,00 & 59,50 & 77,30 & 1,70 & 50,78 & 203,10 & 3,56 & 1,21 & 9,43 & 17,75 & 362,50 & 2146,09 & 2991,92 & 27,44 \\
\hline Total & 10,97 & 235,85 & 304,05 & 269,29 & 3,70 & 252,81 & 1018,26 & 33,45 & 19,11 & 20,43 & 127,56 & 1914,75 & 6692,90 & 10903,14 & \\
\hline$\%$ & 0,10 & 2,16 & 2,79 & 2,47 & 0,03 & 2,32 & 9,34 & 0,31 & 0,18 & 0,19 & 1,17 & 17,56 & 61,39 & & 100,00 \\
\hline
\end{tabular}

Na Tabela 4.5, observa-se o resultado do Resíduo de Limpeza, por estação do ano.

Tabela 4.5 Resíduo de Limpeza gerado na UNICAMP, por estação do ano (kg)

No Resíduo de Limpeza destacou-se a grande quantidade de resíduo de varrição, com 61,4\% do total, e do não reciclável, com 17,6\% do total. Os destaques no resíduo reciclável ficaram para o plástico com 9,4\% e para o papel com 7,5\% do total. Observa-se, na Tabela 4.5, a homogeneidade na distribuição, com certa elevação do percentual no inverno e na primavera, sobretudo, em razão do aumento do item varrição.

Na Tabela. 4.6, pode-se observar a distribuição do Resíduo de Limpeza entre as semanas de caracterização e a que semana do mês se refere. Para tal efetuou-se a distribuição por categorias de massa $(\mathrm{kg})$, variando em $100 \mathrm{~kg}$ cada categoria, aproximadamente $1 \%$ do total de resíduo caracterizado.

\begin{tabular}{|c|c|c|c|}
\hline \multicolumn{2}{|c|}{ Categorias (kg) } & \multirow{2}{*}{$\begin{array}{c}\text { Semana de caracterização } \\
37\end{array}$} & \multirow{2}{*}{$\frac{\text { Semana do mês }}{2^{\mathrm{a}}}$} \\
\hline 1 & $301-400$ & & \\
\hline 2 & $401-500$ & $7,17,33$, e 56 & $3^{\frac{\mathrm{a}}{a}}, 4^{\mathrm{a}}, 3^{\mathrm{a}}$ e $4^{\mathrm{a}}$ \\
\hline 3 & $501-600$ & 23 e 39 & $1^{\mathrm{a}} \mathrm{e} 4^{\mathrm{a}}$ \\
\hline 4 & $601-700$ & 13 & $5^{\mathrm{a}}$ \\
\hline 5 & $701-800$ & $42,45,10$ e 20 & $3^{\underline{\mathrm{a}}}, 2^{\mathrm{a}}, 2^{\underline{\mathrm{a}}}$ e $3^{\mathrm{a}}$ \\
\hline 6 & $801-900$ & & \\
\hline 7 & $901-1000$ & $5,48,27$ & $1^{\mathrm{a}}, 5^{\mathrm{a}}$ e $1^{\mathrm{a}}$ \\
\hline 8 & $1001-1100$ & 34 & $4^{\mathrm{a}}$ \\
\hline
\end{tabular}

Tabela 4.6 Distribuição do Resíduo de Limpeza do Campus Cidade Universitária “Zeferino Vaz" da UNICAMP, por categorias

Verifica-se na Tabela 4.6, que a média de geração nas semanas $(681,45 \mathrm{~kg})$, esteve na 4a categoria, onde se tem: uma 5a semana. Abaixo da categoria da média, teve-se 7 semanas, sendo: uma 1a, uma 2a, duas 3a e três 4a semana(s). Acima da categoria da média, 8 semanas, sendo: duas 1a, duas 2a, duas 3a, uma 4a e uma 5a semana(s). Quando se analisa a influência de fatores externos neste resíduo, considerar-se-ia, principalmente, a influência do período de pagamento, que se encontra na 1a ou 2a semana, o que foi comprovado, com 66,6\% das 1a e 2a semanas acima da média de geração do resíduo.

\subsubsection{Resíduo de Conservação e Limpeza}

O resíduo de Conservação e Limpeza (Tabela 4.7) é composto pela somatória dos valores do resíduo de feira com o resíduo de limpeza.

\begin{tabular}{|c|c|c|c|c|c|c|c|c|c|c|c|c|c|c|c|}
\hline Estação & $\begin{array}{ll}\mathrm{Pp} & \mathrm{bco} \\
\text { reutilizável }\end{array}$ & Pp bco & Pp misto & Papelão & Jornal & Vidro & Plástico & Metal & Ferro & Fraldas & Patogênico & ñ reciclável & Varrição & Total & $\%$ \\
\hline Verão & 1,06 & 71,32 & 112,40 & 78,59 & 2,50 & 74,24 & 365,06 & 13,06 & 18,88 & 43,80 & 57,18 & 719,86 & 1452,11 & 3010,06 & 25,43 \\
\hline Outono & 9,11 & 72,83 & 99,85 & 66,65 & 0,90 & 86,71 & 333,60 & 15,77 & 2,21 & 0,90 & 35,75 & 676,13 & \begin{tabular}{|l|}
1186,76 \\
\end{tabular} & 2587,16 & 21,85 \\
\hline Inverno & 0,80 & 68,5 & 71,80 & 59,15 & 0,00 & 48,40 & 220,30 & 3,085 & 0,00 & 8,3 & 16,88 & 476,30 & \begin{tabular}{|l|}
2054,72 \\
\end{tabular} & 3028,24 & 25,58 \\
\hline Primavera & 0,00 & 73,90 & 64,70 & 81,50 & 2,60 & 55,38 & 252,10 & 3,88 & 1,21 & 9,43 & 17,75 & 504,20 & 2146,09 & 3212,74 & 27,14 \\
\hline Total & 10,97 & 286,55 & 348,75 & 285,89 & 6,00 & 264,73 & 1171,06 & 35,79 & 22,29 & 62,43 & 127,56 & 2376,49 & 6839,68 & 11838,19 & \\
\hline$\%$ & 0,09 & 2,42 & 2,95 & 2,41 & 0,05 & 2,24 & 9,89 & 0,30 & 0,19 & 0,53 & 1,08 & 20,07 & 57,78 & & 100,00 \\
\hline
\end{tabular}


Tabela 4.7 Resíduo de Conservação e Limpeza, gerado no Campus Cidade Universitária "Zeferino Vaz" da UNICAMP, nas estações do ano (kg)

Nota-se neste resíduo grande percentual de varrição. Isto era esperado, pois se trata de um resíduo típico da atividade de limpeza urbana do Campus. Destaca-se no resíduo com potencial de patogenicidade (patogênico e fraldas), a grande quantidade de fraldas descartáveis, sobretudo na Área de Saúde. Este item, somado à grande quantidade de marmitex e restos de alimentos encontrados, também nesta área, pode indicar a necessidade da construção de um local para que as pessoas, que aguardam suas consultas, possam se trocar, alimentar e repousar. Entre o resíduo reciclável, que é $20,54 \%$ deste resíduo, destacam-se o plástico e o papel (branco reutilizável, branco, misto e jornal).

Para se avaliar o potencial de resíduo reciclável contido nas lixeiras do Campus Cidade Universitária “Zeferino Vaz” UNICAMP, elaborou-se a Tabela 4.8, na qual se excluiu a varrição do Resíduo de Conservação e Limpeza.

\begin{tabular}{c|c|c|c|c|c|c|c|c|c|c}
\hline & $\begin{array}{l}\text { Pp bco } \\
\text { Local }\end{array}$ & Po bco & Pp misto & Papelão & Jornal & Vidro & Plástico & Metal & Ferro & Total \\
\hline Área de Saúde & 0,80 & 43,80 & 56,90 & 66,85 & 0,00 & 20,99 & 278,08 & 8,49 & 2,98 & 478,90 \\
\hline Básico & 1,75 & 88,60 & 124,60 & 102,39 & 3,00 & 166,24 & 396,40 & 9,88 & 11,51 & 904,37 \\
\hline Reitoria & 8,42 & 103,45 & 122,55 & 100,05 & 0,70 & 65,58 & 343,78 & 15,09 & 4,63 & 764,24 \\
\hline Feiras & 0,00 & 50,70 & 44,70 & 16,60 & 2,30 & 11,92 & 152,80 & 2,34 & 3,19 & 284,54 \\
\hline Total & 10,97 & $\mathbf{2 8 6 , 5 5}$ & $\mathbf{3 4 8 , 7 5}$ & $\mathbf{2 8 5 , 8 9}$ & $\mathbf{6 , 0 0}$ & $\mathbf{2 6 4 , 7 3}$ & $\mathbf{1 1 7 1 , 0 6}$ & $\mathbf{3 5 , 7 9}$ & $\mathbf{2 2 , 3 0}$ & $\mathbf{2 4 3 2 , 0 4}$ \\
\hline$\%$ & 0,45 & 11,78 & 14,34 & 11,75 & 0,25 & 10,89 & 48,15 & 1,47 & 0,92 & 100,00 \\
\hline
\end{tabular}

Tabela 4.8 Material reciclável contido Resíduo de Conservação e Limpeza, excluindo varrição, acondicionado nas lixeiras do Campus Campinas da UNICAMP (kg)

O material reciclável acondicionado nas lixeiras foi 48,7\% do total do resíduo presente nas mesmas. Destacaram-se o plástico com 48,2\%, o papel (branco reutilizável, branco, misto, papelão e jornal) com $13,6 \%$ e o vidro com $10,9 \%$. Este resultado justifica a implantação de coleta seletiva nas áreas comuns do Campus, atividade que ainda não é desenvolvida.

\subsection{Programa de minimização do resíduo sólido domés- tico da FEC.}

Estes resultados estão divididos em: implantação do programa de minimização de resíduo sólido doméstico da FEC; e, eficiência do programa de minimização.

4.2.1 Implantação do programa de minimização do resíduo sólido doméstico da FEC

Em junho de 2005 iniciou-se o processo de caracterização e análise do potencial de minimização do resíduo gerado na Faculdade. Estas caracterizações foram realizadas até janeiro de 2006, formando um banco de dados para subsidiar as ações que seriam desenvolvidas no programa.

$\mathrm{Na}$ fase de planejamento foram realizadas reuniões com a Comissão de Resíduo Sólido (CRSFEC) e com a Diretoria da FEC para que o projeto tivesse aprovação e apoio legal destas entidades.

Ao final das doze caracterizações concluiu-se que o resíduo gerado na FEC apresentava um significante poten- cial de minimização, principalmente, em relação à redução da quantidade de papel e à quantidade de material descartada de maneira incorreta na coleta seletiva.

Definiu-se que as práticas de minimização a serem apresentadas e adotadas pela comunidade da FEC seriam: utilização da frente e verso das folhas; revisão os textos na tela do computador; redução o tamanho das folhas para recados; utilização de cadernos e blocos até o fim antes de descartá-los; reutilização de envelopes; priorização da comunicação via correio eletrônico; reutilização de clips; utilização apenas da quantidade de papel toalha necessária para secar as mãos; utilização de materiais reaproveitável, reciclável e reciclado; e, destinação de forma correta o resíduo para coleta seletiva da FEC.

$\mathrm{Na}$ implantação do programa foram realizadas as seguintes atividades: palestras e reuniões com alunos e funcionários, destacando os resultados das primeiras caracterizações. Estas palestras contaram com a presença de membros da Comissão de Resíduo Sólido da FEC; envio de e-mails a todos os professores da faculdade destacando os mesmos assuntos tratados com os alunos e funcionários; solicitação à Diretoria da FEC para criação de duas Portarias, uma ressaltando a importância do engajamento de todos no programa de minimização e outra obrigando a cotação de material reciclado em todas as compras da Faculdade. Esta solicitação foi atendida com a criação das Portarias FEC 31/06 e FEC 32/06; atualização semestral do "site" da CRSFEC; esclarecimento de dúvidas sobre o descarte de material para a coleta seletiva; reuniões e conversas constantes com a equipe de limpeza; distribuição de caixas para armazenamento de papel rascunho; e, compra e distribuição de copos plásticos não descartáveis para todos os funcionários e professores da FEC.

Após a implantação do programa foram realizadas novas

\section{reu}

par 


\section{artigos técnicos}

reuniões com professores e funcionários para mostrar os resultados alcançados e novamente destacar a importância de todos para a eficiência do programa.

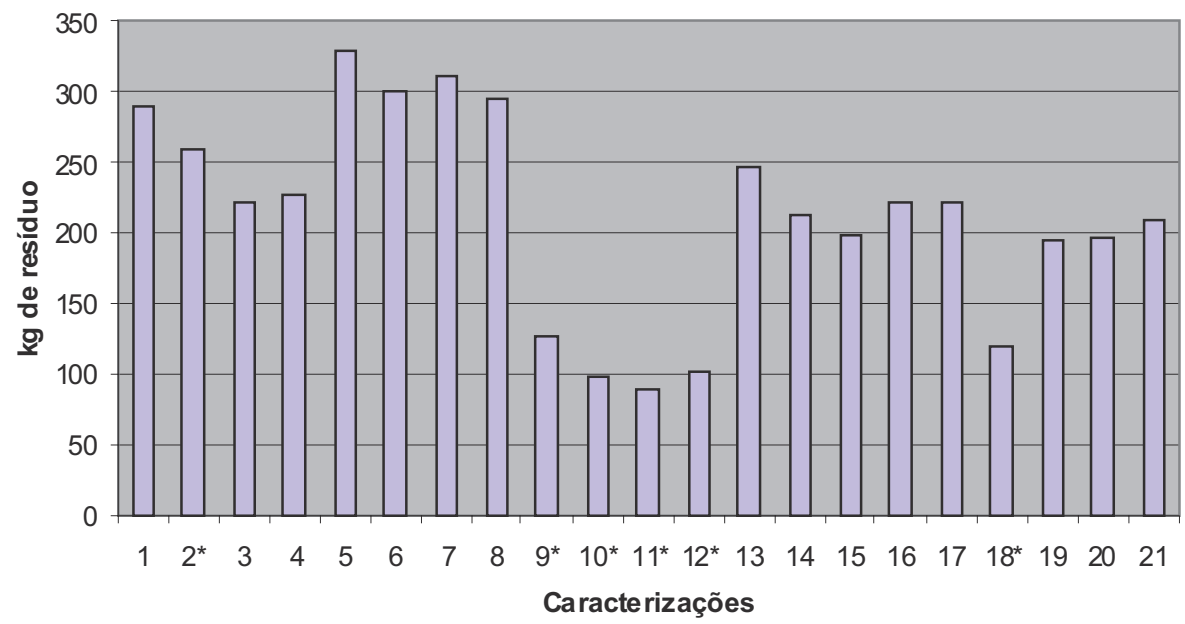

Figura 4.1 Variação na quantidade de resíduo gerada na FEC durante as caracterizações

\subsubsection{Eficiência do Programa de Minimização da FEC}

A quantidade de resíduo gerada na FEC variou consideravelmente nas semanas analisadas, como se observa na Figura 4.1.

Obs.: * caracterização realizada em período de férias escolares. Ressalta-se que as caracterizações de 1 a 12 foram realizadas antes da implantação do programa de minimização e as caracterizações de 13 a 21, depois. Na Figura 4.1 nota-se a grande redução na geração de resíduo sólido doméstico no período de férias de verão. Nesta época a Faculdade é pouco frequentada e parte das suas atividades não acontecem. Nas férias de inverno observa-se pequena variação na quantidade de resíduo gerada na caracterização 2 , pois neste período foram realizadas limpeza em setores e salas da FEC, o que pode ter levado a um aumento da geração de resíduo no período.

Na Figura 4.1 também se observa que houve redução na quan-

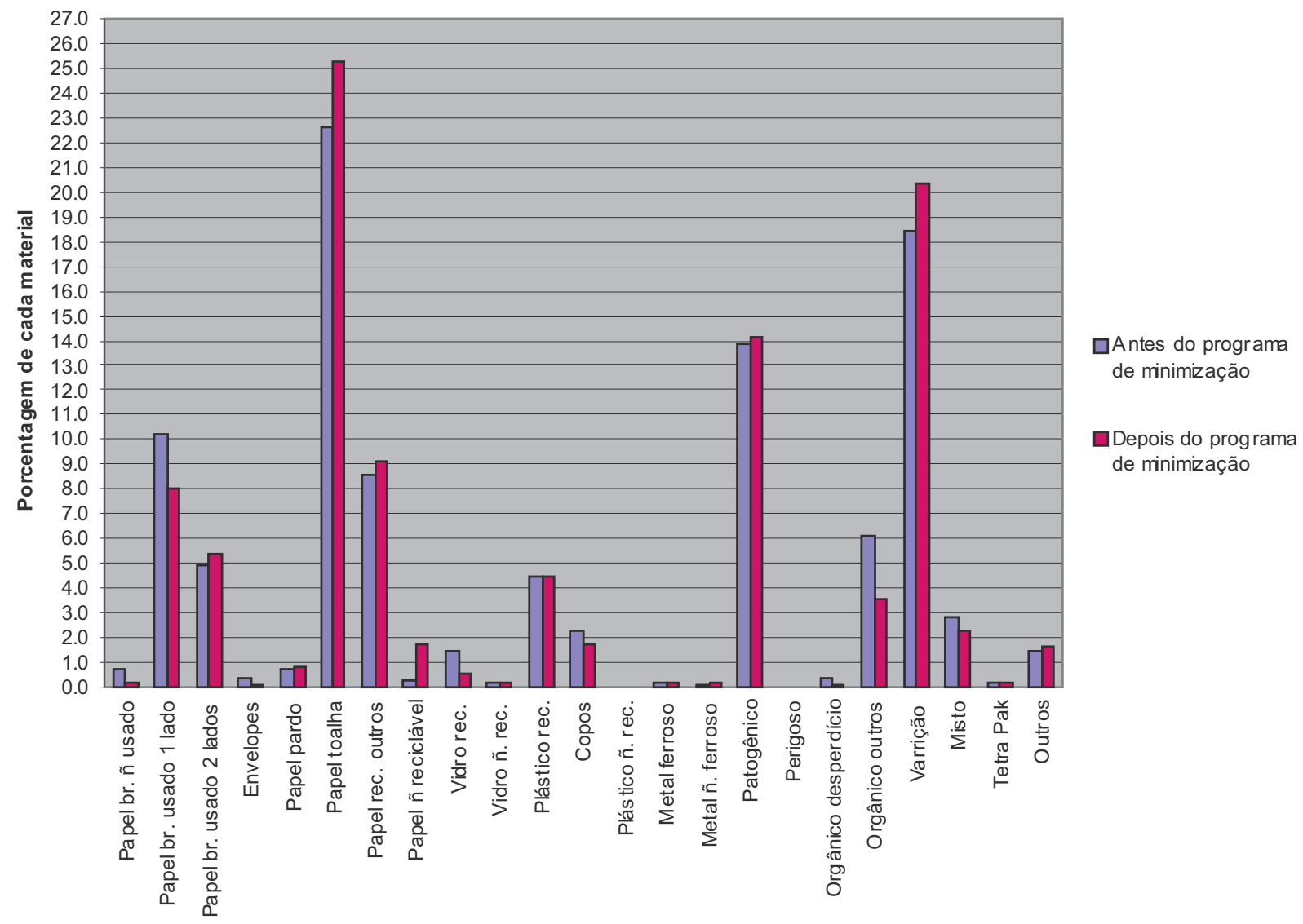

Figura 4.2 Variação da composição do resíduo sólido doméstico gerado na FEC antes e após a implantação doprograma deminimização. 


\section{- artigos técnicos}

tidade de resíduo gerada após a implantação do programa de minimização. A geração média semanal antes da implantação do programa era de $221 \mathrm{~kg}$ e após foi de $201 \mathrm{~kg}$.

A composição percentual do resíduo gerado antes e após o programa é mostrada na Figura 4.2.

Por meio da Figura 4.2 pode-se observar que alguns materiais apresentaram redução na geração. $\mathrm{O}$ que pode indicar que práticas de minimização foram adotadas pela comunidade FEC.

Por outro lado, a geração de papel toalha e de papel branco escrito dos dois lados, aumentou. No caso do papel toalha isto pode ter ocorrido devido à piora na qualidade do papel, o que pode ter levado à utilização de mais folhas para secar as mãos. Já em relação ao papel branco utilizado dos dois lados, o aumento pode indicar que as pessoas estão passando a usar mais a frente e o verso das folhas.

Os demais materiais não foram contemplados nas práticas do programa, apesar de alguns serem passíveis de redução, como o material orgânico e varrição, que poderiam ser compostados e utilizados nas áreas verdes da Faculdade, mas esta atividade não foi possível devido à falta de funcionário para sua realização.

Pode-se perceber também, na Figura 4.2, que tanto antes quanto depois da implantação do programa de minimização, que o papel (somados todos os tipos) representa a maior parte do resíduo sólido doméstico gerado na FEC, seguido de varrição e patogênico.

Analisando a composição do resíduo de papel, observa-se que este é composto em sua maior parte por papel toalha, seguido de papel branco utilizado de apenas um lado e de papel reciclável outros.

\section{Conclusões}

Com base nos dados apresentados, conclui-se que as caracterizações realizadas foram essenciais para fornecer subsídios às ações propostas.

No caso do resíduo gerado nas áreas comuns da UNICAMP, tornou-se evidente que é de grande importância a implantação da coleta seletiva, destacando a quantidade significativa de plástico, papéis (branco, misto e papelão) e vidro coletada. Com relação à presença de papel branco reutilizável, observa-se que o percentual deste material é mínimo, não justificando a implantação de programas de redução ou reutilização para este material nestas áreas.

Já no resíduo gerado na Unidade de Ensino (FEC), o item papel apresentou grande potencial de minimização, sendo, então contemplado no programa. Em relação ao Programa de Minimização de Resíduo Sólido Doméstico, este apresentou bons resultados. Notou-se a redução de papel em branco não usado e de envelopes em condições de reutilização descartados como resíduo. Ainda, observou-se o aumento da geração de papel branco escrito dos dois lados, mostrando o uso mais eficiente deste tipo de material. Assim, conclui-se que é importante a implantação e aumento da abrangência de programas que contemplem a questão do resíduo sólido envolvendo a comunidade escolar. E recomenda-se que as caracterizações nas áreas comuns da UNICAMP e na FEC continuem a ser realizadas.

\section{Referências}

BARBOSA, M. Minimização de resíduo sólido doméstico na Faculdade de Engenharia Civil, Arquitetura e Urbanismo da UNICAMP. 2007. Dissertação (Mestrado em Engenharia Civil). Faculdade de Engenharia Civil, Arquitetura e Urbanismo, Universidade Estadual de Campinas, Campinas, 2007.

HENRIQUE, J. B. de C. Diagnóstico dos serviços de limpeza urbana do Campus Cidade Universitária “Zeferino Vaz” da Universidade Estadual de Campinas. 2008. Dissertação (Mestrado em Engenharia Civil). Faculdade de Engenharia Civil, Arquitetura e Urbanismo, Universidade Estadual de Campinas, Campinas, 2008. 